\title{
Lichens of the Sirumalai hills, Eastern Ghats with one genus and six species new to India
}

\author{
Nayaka $S^{1^{*}}$, Joseph $S^{1}$, Rajaram $S^{2}$, Natesan $S^{2}$, Sankar $K^{2}$, David $M L R^{2}$ and \\ Upreti DK ${ }^{2}$
}

\author{
${ }^{1}$ Lichenology Laboratory, CSIR-National Botanical Research Institute, Rana Pratap Marg, Lucknow-226 001, Uttar \\ Pradesh, India \\ ${ }^{2}$ Department of Biotechnology, Kamaraj College of Engineering and Technology, K. Vellakulam, Near Virudhunagar, \\ Madurai-625 701, Tamil Nadu, India
}

Nayaka S, Joseph S, Rajaram SK, Natesan S, Sankar K, David MLR, Upreti DK 2021 - Lichens of the Sirumalai hills, Eastern Ghats with one genus and six species new to India. Studies in Fungi 6(1), 204-212, Doi 10.5943/sif/6/1/13

\begin{abstract}
Lichens of Sirumalai hills are reported here for the first time. Lichen biota comprised of 95 species. The genus Japewiella is reported for the first time in India and is represented by $J$. tavaresiana (H. Magn.) Printzen. Furthermore, the following six taxa including one variety are new to India viz. Arthonia atra (Pers.) A. Schneid., Graphis brevicarpa M. Nakan., Kashiw. \& K.H. Moon, Micarea erratica (Körb.) Hertel, Rambold \& Pietschm., Pertusaria cicatricosa var. deficiens A.W. Archer, Elix \& Streimam, Porina subargillacea Müll Arg., and Pyxine schmidtii Vain. Brief accounts for all the new records to India are provided to facilitate their identification. Arthonia redingeri Grube and Lepraria caesiella R.C. Harris are reported for the first time from south India. Besides all above, 29 species are recorded for the first time from the state of Tamil Nadu. The lichen biota of the area is primarily corticolous in habitats and dominated by crustose form.
\end{abstract}

Keywords - lichenized fungi - new records - south India - Tamil Nadu - tropical lichens

\section{Introduction}

India supports high lichen biodiversity, with 2714 recorded species. As new areas have been explored as well as revisionary studies in the recent years have resulted in a steep rise in novel species being discovered. During the years 2010 to 2017, a total of 411 species were added to the recorded Indian lichen biota (Sinha et al. 2018). However, many natural habitats of scientific interest remain to be explored. Although, the Eastern Ghats contribute significantly to the species richness and number of endemic lichen species, it has received less attention for conservation than the Western Ghats (Muthumperumal \& Parthasarathy 2010). Nayaka et al. (2013) emphasized the current extent of lichen research in the Eastern Ghats. The lichen survey in Horsley and Tirumala Hills in Andhra Pradesh and Shevaroy Hills in Tamil Nadu resulted in several new additions to the recorded species of the Eastern Ghats (Nayaka et al. 2013). In our ongoing effort to explore new and under explored areas of the country, an attempt to examine the lichen diversity of Sirumalai hills in south India was carried out as a part of the "Workshop on lichen biology and bioprospecting (LBB2017)".

The Sirumalai hills are situated in the southern part of Eastern Ghats in Dindigul district of Tamil Nadu $\left(10^{\circ} .07^{\prime}-10^{\circ} .18^{\prime} \mathrm{N}\right.$ and $\left.77^{\circ} .55^{\prime}-78^{\circ} .12^{\prime} \mathrm{E}\right)$ (Fig. 1). The months March to July are the 
hottest in the hills and temperature range between $28^{\circ} \mathrm{C}$ to $40^{\circ} \mathrm{C}$. The temperature drops significantly during winter and range between $10^{\circ} \mathrm{C}$ to $18^{\circ} \mathrm{C}$. The months from February to August are mostly dry with occasional showers in April and May. There is a little rainfall in September, followed by the heavy showers of the north-eastern monsoon from the middle of October till the end of December. The average annual rainfall in the region is 120 to $132 \mathrm{~cm}$, while the relative humidity varies from $30 \%$ to $80 \%$ (Karuppusamy 2007, Santharam et al. 2014). The area is characterized by disturbed scrub forests cover on lower hills, the dry deciduous forest at midelevation, and semi-evergreen forests at the higher elevation. The slopes of these hills can also be covered by savannah grassland. There have been frequent studies in this region on higher plants, including both medicinal and ethnobotanical plants. Sankar et al. (2009) reported a total of 85 plant species as new records for the Sirumalai hills, while Karuppusamy (2007) listed 90 species of medicinal plants used mainly by the 'Paliyan' tribes dwelling in the area. The altitudinal gradient, rich flora and fauna in the Sirumalai hills, indicates the possibilities of harbouring the luxuriant growth of lichens. Therefore, we surveyed this area to document its anticipated high lichen diversity.

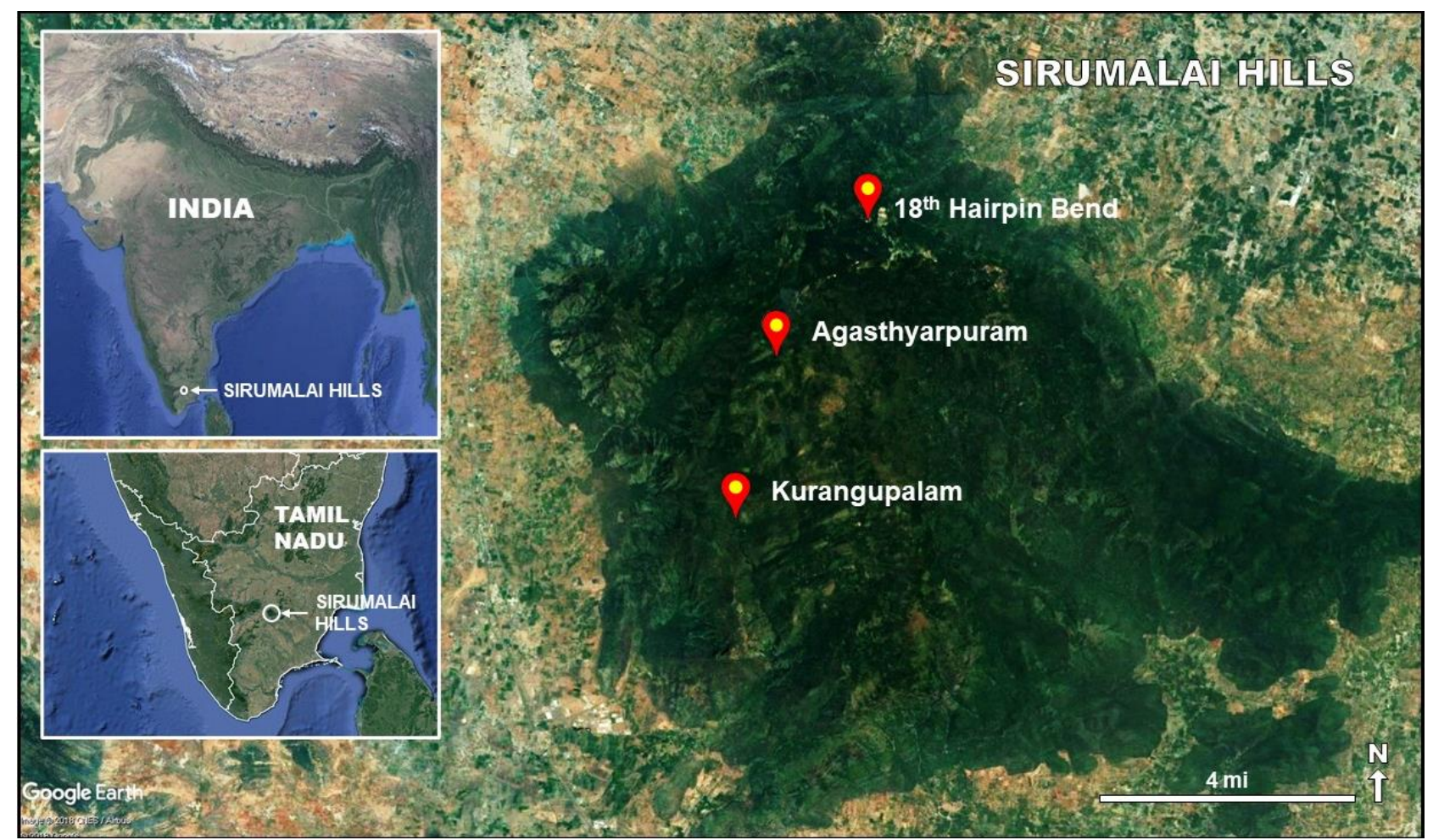

Fig. 1 - Map showing the lichen collection sites in Sirumalai hills. The top left panel of the map shows the location of Sirumalai hills within India. The bottom left panel is the enlarged portion of southern India showing the location of Tamil Nadu state and Sirumalai hills.

\section{Materials \& methods}

A field survey was conducted during December 2017 in the Sirumlai hills and we collected about 200 lichen specimens from three localities viz., Agasthyarpuram $\left(10^{\circ} 22^{\prime} 07.72^{\prime \prime} \mathrm{N}\right.$; $\left.77^{\circ} 37^{\prime} 63.02^{\prime \prime} \mathrm{E}\right)$, Kurangupalam $\left(10^{\circ} 18^{\prime} 56.31^{\prime \prime} \mathrm{N} ; 7^{\circ} 37^{\prime} 76.20^{\prime \prime} \mathrm{E}\right)$ and near $18^{\text {th }}$ Hairpin Bend $\left(10^{\circ} 25^{\prime} 00.87^{\prime \prime} \mathrm{N} ; 7^{\circ} 39^{\prime} 67.25^{\prime \prime} \mathrm{E}\right)$ (Fig. 1). The specimens were identified at CSIR-National Botanical Research Institute, Lucknow and deposited in herbarium LWG. The morphological details were examined using a stereo zoom Leica S8APO microscope. Anatomical details were studied using DM2500 optical microscopes attached with camera and image analysis software. Thin hand-cut sections of thalli and ascomata were mounted in either distilled water, $\mathrm{KOH}$ solution, or lactophenol cotton blue (LPCB). The amyloid reactions were tested using Lugol's iodine solution without or with pre-treatment with $\mathrm{K}$. All measurements were made on material mounted in distilled water. 
Thallus chemistry was studied by spot tests and thin layer chromatography, which was performed in solvent system A and C following Orange et al. (2001). Awasthi (1991, 2007) and several other recent publications were consulted for lichen species identification (Harris 1995, Marbach 2000, McCarthy 2001, Kalb et al. 2004, Nayaka 2005, Coppins 2009, Ertz et al. 2009, Lücking 2009, Saag et al. 2009, Mishra et al. 2011, Aptroot 2012, Allen \& Lendemer 2015, Aptroot \& Lücking 2016, Joseph et al. 2018). Lücking et al. (2017) was followed for the classification of lichens while Jayasiri et al. (2015) was followed to obtain facesoffungi numbers.

\section{Results}

Lichen specimens were identified as belonging to 95 species under 49 genera and 23 families (Table 1). The genus Japewiella is being reported for the first time from India which is represented by species $J$. tavaresiana (H. Magn.) Printzen. Further, six more species of lichens are also being reported for the first time for India viz., Arthonia atra (Pers.) A. Schneid., Graphis brevicarpa M. Nakan., Kashiw. \& K.H. Moon, Micarea erratica (Körb.) Hertel, Rambold \& Pietschm., Pertusaria cicatricosa var. deficiens A.W. Archer, Elix \& Streimam, Porina subargillacea Müll Arg., and Pyxine schmidtii Vain.

The lichen biota of Sirumalai hills is dominated by crustose lichens (54 spp.) followed by foliose (28 spp.), leprose (5 spp.), fruticose (4 spp.) and squamulose (2 spp.) lichens. Similarly, most of the species were corticolous while Cratiria lauri-cassiae (Fée) Marbach, Micarea erratica (Körb.) Hertel, Rambold \& Pietschm., Rinodina oxydata (A. Massal.) A. Massal. and Xanthoparmelia congensis (J. Steiner) Hale are found as saxicolous. Further, Lepraria coriensis (Hue) Sipman was found growing both on soil and rock. Cladonia cartilaginea Müll. Arg. was found exclusively on the soil. The family Parmeliaceae is the most diverse group in the Sirumalai hills with 17 species under eight genera. Graphidaceae is the most dominant family with 18 species under four genera. Among the various genera Graphis is the most dominant genus with 12 species followed by Parmotrema and Pyxine with six species each. The lichen community is also represented a good diversity of pyrenocarpous lichens represented by genera Anisomeridium, Anthracothecium, Lithothelium, Polymeridium, Porina, Pyrenula and Trypethelium. Although several specimens of Lithothelium were collected, we were unable to detect any ascospores. The most common lichens in the area are Dirinaria consimilis (Stirt.) D.D. Awasthi, Heterodermia dissecta (Taylor) D.D. Awasthi, Lecanora helva Stizenb. and Parmotrema tinctorum (Despr. ex Nyl.) Hale.

Previously the lichen biota of the state of Tamil Nadu comprised about 870 species. The present study has added seven new records for India, two new species for south India (Arthonia redingeri Grube and Lepraria caesiella R.C. Harris), and 29 species new to Tamil Nadu [marked as the asterisk $(*)$ in Table 1].

Table 1 List of lichens recorded in the Sirumalai hills

\begin{tabular}{|c|c|c|c|c|c|}
\hline $\begin{array}{l}\text { Sl. } \\
\text { No. }\end{array}$ & Lichen taxa & Family & $\begin{array}{l}\text { Growth } \\
\text { Form }\end{array}$ & Substratum & $\begin{array}{l}\text { Voucher } \\
\text { number }\end{array}$ \\
\hline 1 & *Alyxoria varia (Pers.) Ertz \& Tehler & Lecanographaceae & $\mathrm{CR}$ & Bark & $17-030478$ \\
\hline 2 & $\begin{array}{l}\text { Allographa hossei (Vain.) Lücking \& } \\
\text { Kalb }\end{array}$ & Graphidaceae & $\mathrm{CR}$ & Bark & 17-031125B \\
\hline 3 & $\begin{array}{l}\text { *Anisomeridium biforme (Borrer) R.C. } \\
\text { Harris }\end{array}$ & Monoblastiaceae & $\mathrm{CR}$ & Bark & $\begin{array}{l}\text { 17-030479, } \\
17-031125 \mathrm{~A}\end{array}$ \\
\hline 4 & $\begin{array}{l}\text { Anthracothecium macrosporum (Hepp) } \\
\text { Müll. Arg. }\end{array}$ & Pyrenulaceae & $\mathrm{CR}$ & Bark & $17-030480$ \\
\hline 5 & ${ }^{\circledR}$ Arthonia atra (Pers.) A. Schneid. & Arthoniaceae & $\mathrm{CR}$ & Bark & $17-030453$ \\
\hline 6 & *A. redingeri Grube & Arthoniaceae & CR & Bark & 17-030481A \\
\hline 7 & *A. subvelata $\mathrm{Nyl}$. & Arthoniaceae & $\mathrm{CR}$ & Bark & $17-030482 \mathrm{~A}$ \\
\hline 8 & $\begin{array}{l}\text { *Arthothelium nigrescens Makhija \& } \\
\text { Patw. }\end{array}$ & Arthoniaceae & CR & Bark & $17-030483 \mathrm{C}$ \\
\hline
\end{tabular}


Table 1 Continued.

\begin{tabular}{|c|c|c|c|c|c|}
\hline $\begin{array}{l}\text { Sl. } \\
\text { No. }\end{array}$ & Lichen taxa & Family & $\begin{array}{l}\text { Growth } \\
\text { Form }\end{array}$ & Substratum & $\begin{array}{l}\text { Voucher } \\
\text { number }\end{array}$ \\
\hline 9 & Bacidia millegrana (Taylor) Zahlbr. & Ramalinaceae & $\mathrm{CR}$ & Bark & $17-030484$ \\
\hline 10 & Bulbothrix isidiza (Nyl.) Hale & Parmeliaceae & FL & Bark & $\begin{array}{l}17-030485 \mathrm{~A}, \\
17-031140 \mathrm{~B}\end{array}$ \\
\hline 11 & *B. setschwanensis (Zahlbr.) Hale & Parmeliaceae & FL & Bark & 17-030486A \\
\hline 12 & Caloplaca bassiae (Ach.) Zahlbr. & Teloschistaceae & CR & Bark & $\begin{array}{l}\text { 17-030487, } \\
17-031150 \mathrm{~B}\end{array}$ \\
\hline 13 & *C. herbidella (Arnold) H. Magn. & Teloschistaceae & $\mathrm{CR}$ & Bark & $17-030488$ \\
\hline 14 & *Canomaculina subsumpta (Nyl.) Elix & Parmeliaceae & FL & Bark & 17-030489 \\
\hline 15 & $\begin{array}{l}\text { Canoparmelia texana (Tuck.) Elix \& } \\
\text { Hale }\end{array}$ & Parmeliaceae & FL & Bark & $17-030490$ \\
\hline 16 & $\begin{array}{l}\text { *Chrysothrix chlorina (Ach.) J.R. } \\
\text { Laundon }\end{array}$ & Chrysotrichaceae & LP & Bark & $17-030491$ \\
\hline 17 & Cladonia cartilaginea Müll. Arg. & Cladoniaceae & FR & Soil & $17-030492$ \\
\hline 18 & Coenogonium sp. & Coenogoniaceae & CR & Bark & 17-030493B \\
\hline 19 & Cratiria lauri-cassiae (Fée) Marbach & Caliciaceae & $\mathrm{CR}$ & Rock & $\begin{array}{l}17-030486 \mathrm{C}, \\
17-030495 \mathrm{~B}\end{array}$ \\
\hline 20 & C. obscurior (Stirt.) Marbach \& Kalb & Caliciaceae & $\mathrm{CR}$ & Bark & $\begin{array}{l}\text { 17-030494B, } \\
17-031158 \mathrm{~B}\end{array}$ \\
\hline 21 & $\begin{array}{l}\text { *Cryptothecia polymorpha Makhija \& } \\
\text { Patw. }\end{array}$ & Arthoniaceae & $\mathrm{CR}$ & Bark & $17-030495 \mathrm{~A}$ \\
\hline 22 & $\begin{array}{l}\text { Diorygma hieroglyphicum (Pers.) } \\
\text { Staiger \& Kalb }\end{array}$ & Graphidaceae & $\mathrm{CR}$ & Bark & $17-030496$ \\
\hline 23 & D. junghuhnii (Mont. \& Bosch) Kalb & Graphidaceae & $\mathrm{CR}$ & Bark & 17-030483B \\
\hline 24 & $\begin{array}{l}\text { Dirinaria applanata (Fée) D.D. } \\
\text { Awasthi }\end{array}$ & Caliciaceae & FL & Bark & 17-030485B \\
\hline 25 & D. consimilis (Stirt.) D.D. Awasthi & Caliciaceae & FL & Bark & $17-030468 \mathrm{C}$ \\
\hline 26 & Glyphis cicatricosa Ach. & Graphidaceae & CR & Bark & 17-030497A \\
\hline 27 & ${ }^{*}$ G. scyphulifera (Ach.) Staiger & Graphidaceae & CR & Bark & 17-030497B \\
\hline 28 & Graphis ajarekarii Patw. \& C.R. Kulk. & Graphidaceae & CR & Bark & $\begin{array}{l}17-030469 \mathrm{C}, \\
17-0304671\end{array}$ \\
\hline 29 & *G. argentia Makhija \& Adaw. & Graphidaceae & $\mathrm{CR}$ & Bark & 17-030498A \\
\hline 30 & $\begin{array}{l}{ }^{\circledR} G . \text { brevicarpa M. Nakan., Kashiw. \& } \\
\text { K.H. Moon }\end{array}$ & Graphidaceae & $\mathrm{CR}$ & Bark & $17-030464$ \\
\hline 31 & ${ }^{*}$ G. chlorotica A. Massal. & Graphidaceae & CR & Bark & $17-030499$ \\
\hline 32 & G. cincta (Pers.) Aptroot & Graphidaceae & CR & Bark & 17-030486B \\
\hline 33 & G. duplicata Ach. & Graphidaceae & CR & Bark & $\begin{array}{l}\text { 17-030477A, } \\
17-030483 \mathrm{~A}\end{array}$ \\
\hline 34 & *G. elegans (Borrer ex Sm.) Ach. & Graphidaceae & $\mathrm{CR}$ & Bark & 17-030476A \\
\hline 35 & *G. furcate Fée & Graphidaceae & $\mathrm{CR}$ & Bark & $17-030500 \mathrm{~A}$ \\
\hline 36 & *G. handelii Zahlbr. & Graphidaceae & CR & Bark & 17-030481B \\
\hline 37 & $\begin{array}{l}\text { *G. polystriata Makhija, A. Dube, } \\
\text { Adaw. \& Chitale }\end{array}$ & Graphidaceae & $\mathrm{CR}$ & Bark & $17-031126$ \\
\hline 38 & *G. pyrrhocheiloides Zahlbr. & Graphidaceae & CR & Bark & 17-030482B \\
\hline 39 & *Hafellia demutans (Zahlbr.) Pusswald & Caliciaceae & CR & Bark & 17-031127A \\
\hline 40 & $\begin{array}{l}\text { Heterodermia diademata (Taylor) D.D. } \\
\text { Awasthi }\end{array}$ & Physciaceae & FL & Bark & 17-030456B \\
\hline 41 & H. dissecta (Kurok.) D.D. Awasthi & Physciaceae & FL & Bark & $17-031128$ \\
\hline 42 & $\begin{array}{l}\text { H. japonica (M. Satô) Swinscow \& } \\
\text { Krog }\end{array}$ & Physciaceae & FL & Bark & 17-031129A \\
\hline 43 & H. obscurata (Nyl.) Trevis. & Physciaceae & FL & Bark & $17-031130$ \\
\hline 44 & Hypotrachyna exsecta (Taylor) Hale & Parmeliaceae & FL & Bark & $17-031131$ \\
\hline 45 & *H. neodissecta (Hale) Hale & Parmeliaceae & FL & Bark & $17-031132$ \\
\hline 46 & $\begin{array}{l}@ \text { Japewiella tavaresiana (H. Magn.) } \\
\text { Printzen }\end{array}$ & Lecanoraceae & CR & Bark & $17-030462$ \\
\hline 47 & Lecanora albella (Pers.) Ach. & Lecanoraceae & $\mathrm{CR}$ & Bark & 17-031133B \\
\hline
\end{tabular}


Table 1 Continued.

\begin{tabular}{|c|c|c|c|c|c|}
\hline $\begin{array}{l}\text { Sl. } \\
\text { No. }\end{array}$ & Lichen taxa & Family & $\begin{array}{l}\text { Growth } \\
\text { Form }\end{array}$ & Substratum & $\begin{array}{l}\text { Voucher } \\
\text { number }\end{array}$ \\
\hline 48 & L. chlarotera $\mathrm{Nyl}$. & Lecanoraceae & $\mathrm{CR}$ & Bark & 17-030477B \\
\hline 49 & L. helva Stizenb. & Lecanoraceae & $\mathrm{CR}$ & Bark & $\begin{array}{l}17-030468 \mathrm{~B}, \\
17-030482 \mathrm{C}\end{array}$ \\
\hline 50 & L. perplexa Brodo & Lecanoraceae & $\mathrm{CR}$ & Bark & $17-031134$ \\
\hline 51 & Lepraria caesiella R.C. Harris & Stereocaulaceae & LP & Bark & $17-030463$ \\
\hline 52 & $\begin{array}{l}\text { L. caesioalba (B. de Lesd.) J.R. } \\
\text { Laundon }\end{array}$ & Stereocaulaceae & LP & Bark & $17-030455$ \\
\hline 53 & $*$ L. coriensis $(\mathrm{Hue})$ Sipjman & Stereocaulaceae & LP & Rock \& Soil & $17-031135$ \\
\hline 54 & *L. jackii Tønsberg & Stereocaulaceae & LP & Bark & $17-031136$ \\
\hline 55 & $\begin{array}{l}\text { Leptogium austroamericanum (Malme) } \\
\text { C.W. Dodge }\end{array}$ & Collemataceae & FL & Bark & $17-031137$ \\
\hline 56 & Lithothelium sp. & Pyrenulaceae & $\mathrm{CR}$ & Bark & $17-031138$ \\
\hline 57 & $\begin{array}{l}{ }^{@} \text { Micarea erratica (Körb.) Hertel, } \\
\text { Rambold \& Pietschm. }\end{array}$ & Pilocarpaceae & $\mathrm{CR}$ & Rock & $17-030460$ \\
\hline 58 & Ochrolechia subpallescens Verseghy & Ochrolechiaceae & $\mathrm{CR}$ & Bark & 17-030466B \\
\hline 59 & Parmelia erumpens Kurok. & Parmeliaceae & FL & Bark & $17-031139$ \\
\hline 60 & $\begin{array}{l}\text { Parmelinella wallichiana (Taylor) Elix } \\
\& \text { Hale }\end{array}$ & Parmeliaceae & FL & Bark & $\begin{array}{l}\text { 17-030466A, } \\
17-030474 \mathrm{~B}\end{array}$ \\
\hline 61 & Parmotrema crinitum (Ach.) M. Choisy & Parmeliaceae & FL & Bark & $\begin{array}{l}17-030473 \mathrm{~B}, \\
17-030493 \mathrm{~A}\end{array}$ \\
\hline 62 & $* P$. cristiferum (Taylor) Hale & Parmeliaceae & FL & Bark & $17-031140 \mathrm{~A}$ \\
\hline 63 & P. hababianum (Gyeln.) Hale & Parmeliaceae & FL & Bark & $17-031141$ \\
\hline 64 & P. praesorediosum (Nyl.) Hale & Parmeliaceae & FL & Bark & $17-031142$ \\
\hline 65 & P. reticulatum (Taylor) M. Choisy & Parmeliaceae & FL & Bark & $17-031143 \mathrm{~A}$ \\
\hline 66 & P. tinctorum (Despr. ex Nyl.) Hale & Parmeliaceae & FL & Bark & $\begin{array}{l}17-030469 D, \\
17-031144\end{array}$ \\
\hline 67 & $\begin{array}{l}{ }^{@} \text { Pertusaria cicatricosa Müll. Arg. var. } \\
\text { deficiens A.W. Archer, Elix \& } \\
\text { Streimam }\end{array}$ & Pertusariaceae & $\mathrm{CR}$ & Bark & $\begin{array}{l}\text { 17-030451A, } \\
17-030450 \mathrm{~B}\end{array}$ \\
\hline 68 & P. cinchonae Müll. Arg. & Pertusariaceae & $\mathrm{CR}$ & Bark & 17-030476B \\
\hline 69 & P. endoxantha Vain. & Pertusariaceae & $\mathrm{CR}$ & Bark & $17-030473 \mathrm{~A}$ \\
\hline 70 & *P. leucostoma (Ach.) A. Massal. & Pertusariaceae & $\mathrm{CR}$ & Bark & $17-030494 \mathrm{C}$ \\
\hline 71 & $\begin{array}{l}\text { P. splendens D.D. Awasthi \& Preeti } \\
\text { Srivast. }\end{array}$ & Pertusariaceae & $\mathrm{CR}$ & Bark & $17-031145 \mathrm{~A}$ \\
\hline 72 & *Phaeographis caesiodisca Staiger & Graphidaceae & $\mathrm{CR}$ & Bark & $\begin{array}{l}17-030469 \mathrm{~B}, \\
17-030473 \mathrm{D}\end{array}$ \\
\hline 73 & Phyllopsora furfuracea Zahlbr. & Ramalinaceae & SQ & Bark & $17-031146$ \\
\hline 74 & *P. nemoralis Timdal \& Krog & Ramalinaceae & SQ & Bark & $17-030495 \mathrm{C}$ \\
\hline 75 & *Polymeridium refertum (Stirt.) Aptroot & Trypetheliaceae & $\mathrm{CR}$ & Bark & $17-031147$ \\
\hline 76 & @ Porina subargillacea Müll Arg. & Porinaceae & $\mathrm{CR}$ & Bark & $17-030458$ \\
\hline 77 & Pyrenula adacta Fée & Pyrenulaceae & $\mathrm{CR}$ & Bark & $17-031148$ \\
\hline 78 & P. astroidea (Fée) R.C. Harris & Pyrenulaceae & $\mathrm{CR}$ & Bark & $17-031149$ \\
\hline 79 & Pyxine cocoes (Sw.) Nyl. & Caliciaceae & FL & Bark & $17-031150 \mathrm{~A}$ \\
\hline 80 & P. petricola Nyl. & Caliciaceae & FL & Bark & $17-031151$ \\
\hline 81 & *P. philippina Vain. & Caliciaceae & FL & Rock & $17-031152$ \\
\hline 82 & P. reticulata (Vain.) Vain. & Caliciaceae & FL & Bark & $17-031153$ \\
\hline 83 & ${ }^{\circledR} P$. schmidtii Vain. & Caliciaceae & FL & Bark & $\begin{array}{l}17-030457 \mathrm{~A}, \\
17-030475\end{array}$ \\
\hline 84 & P. subcinerea Stirt. & Caliciaceae & FL & Bark & $\begin{array}{l}17-030451 \mathrm{~B}, \\
17-030457 \mathrm{~B}\end{array}$ \\
\hline 85 & Ramalina conduplicans Vain. & Ramalinaceae & FR & Bark & $17-030468 \mathrm{~A}$ \\
\hline 86 & R. pacifica Asahina & Ramalinaceae & FR & Bark & 17-031143B \\
\hline 87 & R. subpusilla (Nyl.) Zahlbr. & Ramalinaceae & FR & Bark & 17-031127B \\
\hline 88 & $\begin{array}{l}\text { Remototrachyna flexilis (Kurok.) } \\
\text { Divakar \& A. Crespo }\end{array}$ & Parmeliaceae & FL & Bark & 17-031129A \\
\hline
\end{tabular}


Table 1 Continued.

\begin{tabular}{|c|c|c|c|c|c|}
\hline $\begin{array}{l}\text { Sl. } \\
\text { No. }\end{array}$ & Lichen taxa & Family & $\begin{array}{l}\text { Growth } \\
\text { Form }\end{array}$ & Substratum & $\begin{array}{l}\text { Voucher } \\
\text { number }\end{array}$ \\
\hline 89 & $\begin{array}{l}\text { Rinodina oxydata (A. Massal.) A. } \\
\text { Massal. }\end{array}$ & Physciaceae & $\mathrm{CR}$ & Rock & $17-031154$ \\
\hline 90 & $\begin{array}{l}\text { Synarthonia inconspicua (Stirt.) Van } \\
\text { den Broeck \& Ertz }\end{array}$ & Arthoniaceae & $\mathrm{CR}$ & Bark & $17-030452$ \\
\hline 91 & Tephromela atra (Huds.) Hafellner & Tephromelataceae & CR & Bark & $17-031155$ \\
\hline 92 & Trypethelium eluteriae Spreng. & Trypetheliaceae & CR & Bark & $17-031156$ \\
\hline 93 & Usnea aciculifera Vain. & Parmeliaceae & FR & Bark & $17-031157$ \\
\hline 94 & $\begin{array}{l}\text { Xanthoparmelia congensis (J. Steiner) } \\
\text { Hale }\end{array}$ & Parmeliaceae & $\mathrm{FL}$ & Rock & $17-031158 \mathrm{~A}$ \\
\hline 95 & $\begin{array}{l}\text { *Zwackhia viridis (Ach.) Poetsch \& } \\
\text { Schied. }\end{array}$ & Lecanographaceae & $\mathrm{CR}$ & Bark & $17-031159$ \\
\hline
\end{tabular}

\section{New Records to India}

Arthonia atra (Pers.) A. Schneid., Guide Study Lich.: 131. 1898

Index Fungorum number: IF376348; Faces of fungi number: FoF09883

Fig. 2A

Thallus crustose, corticolous, whitish grey, ascomata lirellate, in groups, black, exciple carbonized, discontinuous at base, ascospores hyaline, transversely 3-septate, 18.0-25.2 × 4.1-4.5 $\mu \mathrm{m}$.

Remarks - This is a cosmopolitan species found throughout Asia, Africa, Australia, Europe, Macaronesia, North and South America (Pentecost \& James 2009). Previously this species was known as Opegrapha atra Pers., but recently Ertz et al. (2009) reinstated it as Arthonia atra.

Material examined - Nayaka et al. 17-030453 (LWG).

Graphis brevicarpa M. Nakan., Kashiw. \& K.H. Moon, Bull. Nat. Sci. Mus., Tokyo, B 28(4): 107. 2002

Index Fungorum number: IF372560; Faces of fungi number: FoF09882

Fig. 2B

Thallus crustose, corticolous, smooth, whitish grey, apothecia lirellate, immersed, short, mostly unbranched, covered by thick thalline margin up to top, disc concealed, epruinose, exciple laterally carbonized, hymenium inspersed, ascospores transversely septate, 20.8-22.8 $\times 6.3-7.2$ $\mu \mathrm{m}$, and trace amount of norsticitic acid detected in TLC.

Remarks - Previously known from Oceania (Lücking 2009).

Material examined - Nayaka et al. 17-030464 (LWG).

Japewiella tavaresiana (H. Magn.) Printzen, Arnoldia 18: 4. 2000

Index Fungorum number: IF462818; Faces of fungi number: FoF09881

Fig. 2C

Thallus crustose, corticolous, verruculose, greenish to matt, apothecia round, slightly irregular in shape, $0.2-0.5 \mathrm{~mm}$ diam., disc yellowish to cream, margin paler than the disc, thin and persistent at maturity, exciple with radiating hyphae, ascospores hyaline, simple, 9.3-14.9 × 5.6$6.2 \mu \mathrm{m}$, and atranorin (minor) detected in TLC.

Remarks - Earlier, this species was reported from Europe, Macaronesia (James 2009) and the Appalachian Mountains of Eastern North America (Allen \& Lendermer 2015).

Material examined - Nayaka et al. 17-030462 (LWG).

Micarea erratica (Körb.) Hertel, Rambold \& Pietschm., Biblioth. Lichenol. 34: 227. 1989

Index Fungorum number: IF125877; Faces of fungi number: FoF09880

Fig. 2D 
Thallus crustose, saxicolous, smooth, areolate, greenish grey, apothecia small, up to $0.2 \mathrm{~mm}$ diam., round, disc and margin black, epithecium greenish, hymenium and hypothecium hyaline, ascospores haline, narrowly ellipsoid, up to 3 pesudoseptate, 5.8-10.3 $\times 3.6-4.2 \mu \mathrm{m}$, no lichen substances detected in TLC.

Remarks - This species was previously reported from England, Scotland, Ireland, Central Europe, North America, Australia and New Zealand (Coppins 2009). It also occurs on wood. Sirumalai hill specimen slightly differs in having hyaline hypothecium and pseudoseptate ascospores.

Material examined - Nayaka et al. 17-030460 (LWG).
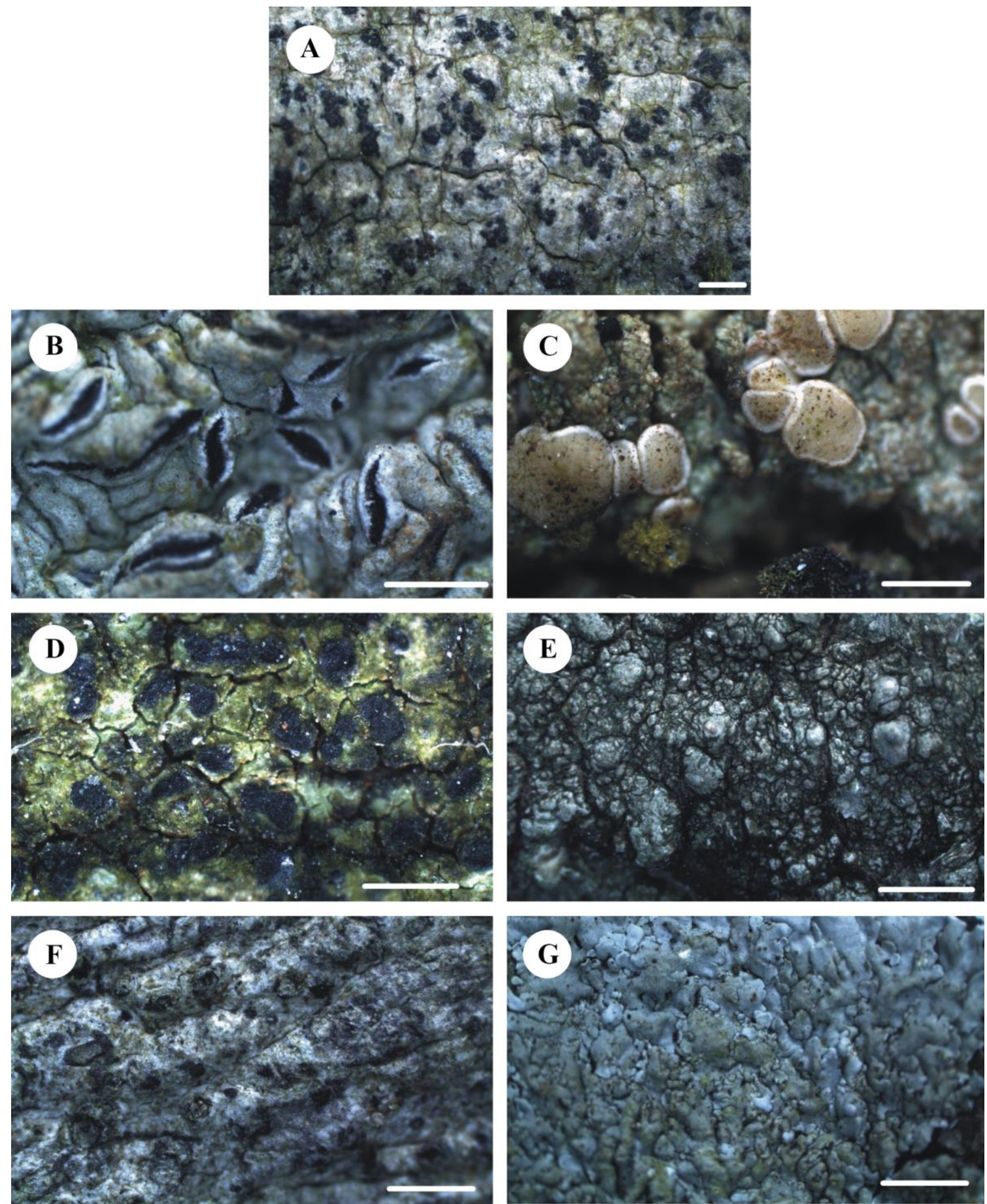

Fig. 2 - New records. A Arthonia atra. B Graphis brevicarpa. C Japewiella tavaresiana. D Micarea erratica. E Pertusaria cicatricosa var. deficiens. F Porina subargillacea. G Pyxine schmidtii. Scale bars: $A=2 \mathrm{~mm}, \mathrm{~B}, \mathrm{C}, \mathrm{D}, \mathrm{E}=1 \mathrm{~mm}, \mathrm{~F}, \mathrm{G}=2 \mathrm{~mm}$. 
Index Fungorum number: IF413724; Faces of fungi number: FoF09879

Thallus corticolous, crustose, greenish grey, cracked, verruculose, apothecia numerous, verruciform, tuberculate, irregular in outline, constricted at base, ostiole inconspicuous, ascospores 2 per ascus, hyaline, ellipsoidal, with prominent rough inner wall, 120.5-184.8 $\times 27.2-49.2 \mu \mathrm{m}$, stictic acid complex detected in TLC.

Remarks - Pertusaria cicatricosa var. deficiens differs from P. cicatricosa var. cicatricosa by lacking chlorinated lichexanthone. This species has previously only been reported from its type locality Papua New Guinea (Archer et al. 1995).

Material examined - Nayaka et al. 17-030451A (LWG).

Porina subargillacea Müll Arg., Bull. Herb. Boissier 1: 64.1893

Fig. 2F

Index Fungorum number: IF402279; Faces of fungi number: FoF09878

Thallus corticolous, crustose, whitish grey, matt, ecorticate, perithecia emergent, black, $0.2-$ $0.4 \mathrm{~mm}$ diam., ostiole inconspicuous, ascospores colourless, transversely 7-septate, fusiform, 33.4$40.8 \times 2.1-2.5 \mu \mathrm{m}$, unidentified triterpenes at $\mathrm{R}_{\mathrm{f}}$ class 6-7 detected in TLC.

Remarks - This species was reported from Australia and New Zealand (McCarthy 2001).

Material examined - Nayaka et al. 17-030458 (LWG).

Pyxine schmidtii Vain., Hedwigia 46: 170. 1907

Index Fungorum number: IF403619; Faces of fungi number: FoF09877

Fig. 2G

Thallus foliose, corticolous, whitish grey, up to $2.5 \mathrm{~cm}$ diam., adnate, lobes imbricate, plane to convex, upper surface pruinose towards lobe tips, pseudocyphellae rare, medulla white, lower surface black at centre, paler towards margin, rizhinate, apothecia absent, atranorin, zeorin, terpenes at $R_{\mathrm{f}}$ class 2 to 3 and at 3 are detected in TLC.

Remarks - Previously this species was reported from Australia, Asia and Papua New Guinea (Elix 2009).

Materials examined - Nayaka et al. 17-030457A, 17-030475 (LWG).

\section{Acknowledgements}

The authors are thankful to the Director of CSIR-NBRI for providing laboratory facilities and to the Principal of Kamaraj College of Engineering and Technology, Head of Biotechnology Department and members of the organizing committee for the LBB2017 workshop. One of the authors (SJ) is thankful to DST, New Delhi for the financial assistance under INSPIRE Faculty scheme (IFA18-LSPA 124). The authors are also grateful to Sirumalai Reserve Forest authorities for their permission to study the lichens of the area. (CSIR-NBRI manuscript numberCSIRNBRI_MS/2019/12/04).

\section{References}

Allen JL, Lendemer C. 2015 - Japewiella dollypartoniana, a new widespread lichen in the Appalachian Mountains of Eastern North America. Castanea 80, 59-65.

Aptroot A. 2012 - A world key to the species of Anthracothecium and Pyrenula. Lichenologist 44, $5-53$.

Aptroot A, Lücking R. 2016 - A revisionary synopsis of the Trypetheliaceae (Ascomycota: Trypetheliales). Lichenologist 48, 763-982.

Archer A, Elix JA, Streimann H. 1995 - The lichen genus Pertusaria (Lichenised Ascomycotina) in Papua New Guinea. Mycotaxon 56, 387-401.

Awasthi DD. 1991 - A key to the microlichens of India, Nepal and Sri Lanka. Bibliotheca Lichenologica 40, 1-336.

Awasthi DD. 2007 - A Compendium of the Macrolichens from India, Nepal and Sri Lanka. Bishen 
Singh Mahendra Pal Singh, Dehra Dun.

Coppins BJ. 2009 - Micarea Fr. Pp 583-606 in CW Smith et al., eds, The Lichens of Great Britain and Ireland. British Lichen Society, London.

Elix JA. 2009 - Pyxine. Pp. 517-533 in PM McCarthy, ed, Flora of Australia. Volume 57, Lichenes

5. CSIRO Publishing, Canberra.

Ertz D, Miadlikowska J, Lutzoni F, Dessein S et al. 2009 - Towards a new classification of the Arthoniales (Ascomycota) based on a three-gene phylogeny focussing on the genus Opegrapha. Mycological Research 113, 141-152.

Harris RC. 1995 - More Florida Lichens. Including the 10ø Tour of the Pyrenolichens. Bronx: Published by the author.

James PW. 2009 - Japewiella Printzen. Pp. 449-450 in CW Smith et al., eds, The Lichens of Great Britain and Ireland. British Lichen Society, London.

Jayasiri SC, Hyde KD, Ariyawansa HA, Bhat DJ, Buyck B, Cai L, Dai Y et al. 2015 - The Faces of Fungi database: fungal names linked with morphology, phylogeny and human impacts. Fungal Diversity 74, 3-18.

Joseph S, Sinha GP, Ramachandran VS. 2018 - Taxonomic Revision of the Lichen Genus Opegrapha sensu lato (Roccellaceae) in India. Indian Journal of Forestry, Additional Serie VI. Bishen Singh Mahendra Pal Singh, Dehradun.

Kalb K, Staiger B, Elix JA. 2004 - A monograph of the lichen genus Diorygma-A first attempt. Symbolae Botanicae Upsalienses 34, 133-181.

Karuppusamy S. 2007 - Medicinal plants used by Paliyan tribes of Sirumalai hills of southern India. Natural Product Radiance 6, 436-442.

Lücking R. 2009 - The taxonomy of the genus Graphis sensu Staiger (Ascomycota: Ostropales: Graphidaceae). Lichenologist 41, 319-362.

Lücking R, Hodkinson BP, Leavitt SD. 2017 - The 2016 classification of lichenized fungi in the Ascomycota and Basidiomycota-Approaching one thousand genera. Bryologist 119, 361-416.

Marbach B. 2000 - Corticole und lignicole Arten der Flechtengattung Buellia sensu lato in den Subtropen und Tropen. Bibliotheca Lichenologica 74, 1-384.

McCarthy PM. 2001 - Trichotheliaceae. Pp 105-157 in PM McCarthy, ed, Flora of Australia. Volume 58A, Lichens 3. CSIRO Publishing, Canberra.

Mishra GK, Upreti DK, Nayaka S, Haridas B. 2011 - New taxa and new reports of Phyllopsora (lichenized Ascomycotina) from India. Mycotaxon115, 29-44.

Muthumperumal C, Parthasarathy N. 2010 - A large-scale inventory of liana diversity in tropical forests of South Eastern Ghats, India. Systematics and Biodiversity 8, 289-300.

Nayaka S. 2005 - Revisionary studies on lichen genus Lecanora sensu lato from India. PhD thesis, Dr. RML Avadh University, Faizabad, India.

Nayaka S, Reddy MA, Ponmurugan P, Anjali DB, Ayyappadasa G. 2013 - Eastern Ghats, biodiversity reserves with unexplored lichen wealth. Current Science 104, 821-825.

Orange A, James PW, White FJ. 2001. Microchemical Methods for the Identification of Lichens. British Lichen Society, London.

Pentecost A, James PW. 2009 - Opegrapha Ach. Pp. 631-647 in CW Smith et al., eds, The Lichens of Great Britain and Ireland. British Lichen Society, London.

Saag L, Saag A, Randlane T. 2009 - World survey of the genus Lepraria (Stereocaulaceae, lichenized Ascomycota). Lichenologist 41, 25-60.

Sankar RV, Kottaimuthu R, Ravikumar K. 2009 - Addition to the flora of Sirumalai hills, Eastern Ghats, India. Journal of Threatened Taxa 1, 379-381.

ISantharam V, Sathasivam K, Badrinarayanan T, Sudhakar KV. 2014 - Birds of the Sirumalai Hills. Indian Birds 9, 57-63.

Sinha GP, Nayaka S, Joseph S. 2018 - Additions to the checklist of Indian lichens after 2010. Cryptogam Biodiversity and Assessment, Special Issue, 197-206. 